\title{
Lycopene supplementation protects vascular dementia gerbils against the impairment of learning and memory
}

\author{
Wei Chen ${ }^{1 \#}$, Jianwu Zhang ${ }^{2}$, Jing Wang ${ }^{3 \#}$, Yunxiang Li ${ }^{4 \#, ~ W e n h u ~}$ Liu $^{2}$, Jun Xia ${ }^{5}$ \\ ${ }^{1}$ Department of Pathophysiology, School of Basic Medical Science, North Sichuan Medical College, Nanchong City, China, \\ ${ }^{2}$ Department of Pharmacology, School of Pharmacy, North Sichuan Medical College, Nanchong City, China, ${ }^{3}$ Pharmacy Department, \\ The People's Hospital of Deyang City, Sichuan province, China, ${ }^{4}$ Urology Department, Nanchong Central Hospital Affiliated to North \\ Sichuan Medical College, Nanchong City, China, ${ }^{5}$ Department of Student Office, School of Clinical Medical Science, North Sichuan \\ Medical College, Nanchong City, China \\ \#Wei Chen, Jing Wang, Yunxiang Li contributed equally to this work.
}

\begin{abstract}
In this study, we investigated the neuroprotective effects of lycopene (Lyc) on vascular dementia (VaD) gerbils and its related mechanisms of anti-inflammatory, anti-apoptotic and anti-oxidant activity. Gerbils were treated with bilateral common carotid arteries. Animals were divided into 1) Sham, 2) VaD model, and 3) VaD model + Lyc $(20 \mathrm{mg} / \mathrm{kg})$ groups. Each group (3) was administered intergalactic Lyc twice a day for 28 days. Morris water mazes were used to evaluate learning and memory ability. Nissl, NeuN, and GFAP staining were used to observe histomorphological changes of neural and glial cells in the hippocampus CA1 region. Western blotting was used to detect hippocampus caspase-3, B-cell lymphoma-2 (BCl-2), BCl-2 related $\times$ protein (Bax), and interleukin (IL)-1, IL-6 and tumour necrosis factor $\alpha$ (TNF- $\alpha$ ) expression amongst other inflammatory factors. Secreted IL-1, IL-6 and TNF- $\alpha$ levels were assessed by ELISA in addition to superoxide dismutase (SOD), glutathione peroxidase (GSH-PX), glutathione (GSH) and malondialdehyde (MDA).

We found that Lyc increased the learning and memory ability of gerbils by reducing the latency time in the Morris water maze, and increasing spatial memory. Lyc also reduced pathological changes in the hippocampus caused by $\mathrm{VaD}$, reduced apoptosis, and decreased VaD mediated BCl-2/Bax expression. The levels of inflammatory factors and oxidative stress were also reduced by Lyc in the VaD models.

We therefore conclude that Lyc can improve the learning and memory ability of VaD gerbils, the mechanism of which may be related to reduced oxidative stress and apoptosis in VaD hippocampus neurons.
\end{abstract}

Key words: lycopene, neuroprotective effects, vascular dementia, anti-oxidant, anti-inflammatory, apoptosis, oxidative stress, learning, memory, hippocampus neurons.

\section{Introduction}

Dementia affects approximately 36 million people globally. Amongst its subtypes, vascular demen- tia (VaD) accounts for up to $20 \%$ of all dementia cases and is the second major cause of dementia in the elderly [41]. VaD results from a range of aetiol-

Communicating author:

Jianwu Zhang, Department of Pharmacology, School of Pharmacy, North Sichuan Medical College, Nanchong City, 637100, China, e-mail: chengwei197959@163.com 
ogies including cerebrovascular dysfunction (CVD). The reported risk factors for VaD include diabetes, insulin resistance, high body mass index (BMI)/ obesity, hypertension and high blood cholesterol $[2,9,19]$. VaD will occur when blood vessels supplying the brain are restricted, leading to a loss of oxygen delivery and hypoxia [30]. The occurrence of $\mathrm{VaD}$ is reduced through diabetes treatment, blood pressure reduction, and lipid lowering therapy $[9,13,16,18-20]$.

Vascular dementia has many early-warning signs including delayed thinking processes, difficulties in planning, attention deficit, mood and concentration behavioural changes [40]. If VaD remains untreated, these symptoms would progressively come to worsen. Despite its importance, there are no FDA-approved treatments which can reverse VaD-mediated damage. Medications for the treatment of other heterogeneous disorders including Alzheimer's disease (AD) provide only modest benefits [3].

Reduced cerebral blood flow leads to oxygen and glucose deprivation. This is followed by neuroinflammation, oxidative stress and nitrosative stress, known causes of $\mathrm{VaD}$ [4]. Low oxidant concentrations elevate the levels of oxygen free radicals and their derivatives, leading to excessive protein nitrosylation and neuronal dysfunction $[1,6-8,10,12,15]$. Studies have shown that oxidative stress is strictly linked to not only the activation of metalloproteinases and proteases involved in proteolytic disruption of the blood brain barrier (BBB), but also in white matter lesions typical of $\mathrm{VaD}[37,38,44-46]$. In $\mathrm{VaD}$, the antioxidant defence is insufficient. VaD patients regularly present with folate deficiency and low vitamin E levels. In addition, superoxide dismutase (SOD) and glutathione (GSH) levels are reduced [14,24,26,32,34-36]. Epidemiological studies indicate that a diet rich in fruit and vegetables decrease the VaD risk [14]. Despite the evidence, it is not clear which individual compounds bring these potential benefits.

Lycopene (Lyc) is a naturally occurring carotenoid pigment found in fruit. Lyc is a photochemical that possesses oxygen quenching activity and participates in many chemical reactions that protect cells from oxidative stress [11]. As a potent antioxidant, Lyc has been shown to afford neuroprotection against microglia activation and focal cerebral ischemia and can attenuate early brain injury following subarachnoid haemorrhage through preventing neuronal apoptosis, maintaining BBB integrity, and decreasing inflammation [17,27]. Lycopene could improve spatial learning and memory impairment by $A \beta_{1-42}$, which suggests that lycopene may be a potential candidate for $A D$ treatment [36]. Despite its known ability to prevent oxidative stress-mediated neuronal death in patients with neurodegeneration, its benefits to $\mathrm{VaD}$ remain largely uncharacteristic.

The purpose of this study was to evaluate the neuroprotective properties of Lyc in a gerbil model of VaD. We hypothesized that Lyc played an important role in improving $\mathrm{VaD}$ by preventing brain impairment and improving learning and memory functions through influencing apoptosis and inflammatory mechanisms.

We examined the effects of Lyc on induced cognitive impairment in the hippocampus of gerbils following bilateral common carotid artery occlusion.

\section{Material and methods \\ Animals and lycopene administration}

Gerbils were purchased from the North Sichuan Medical College Experimental Animal Centre. The study protocol was approved by the Ethical Review Committee of Sichuan Experimental Animal Society. Gerbils weighed 60-80 g. Animals were divided into three groups: 1) Sham group, 2) VaD model, and 3) VaD model + Lyc. Lycopene (Nanjing Zelang Biological Technology Co., Ltd.) was dissolved in 1\% DMSO (Solarbio China) and administered intergalactic Lyc $(20 \mathrm{mg} / \mathrm{kg})$ twice a day for 28 days. Model animals were produced by treatment to the bilateral common carotid arteries. Briefly, gerbils were anesthetized with $10 \%$ chloral hydrate $(350 \mathrm{mg} / \mathrm{kg}$ ). The bilateral common carotid arteries were isolated from the surrounding tissue and vagus nerve and then occluded using artery clips. After $5 \mathrm{~min}$, the clips were removed to restore cerebral blood flow. Complete reperfusion of the arteries was verified by direct visualization and then the neck incision area was sutured. The bilateral common carotid artery and vagus nerve were separated without ligation in the blank group. The body temperature was maintained at $36-37^{\circ} \mathrm{C}$ with a heating pad at all times during this procedure.

All groups contained gerbils $(n=8)$. Random gerbils from each group were selected for learning and memory tests using the Morris water maze. The remaining untested gerbils being sacrificed for other assessments on day 28. 


\section{Behavioural tests}

\section{Morris water maze}

Spatial learning and memory of the VaD gerbils were assessed using the Morris Water Maze (MWM). Briefly, gerbils were trained on 1 day to find the platform that was hidden at a depth of $1.5 \mathrm{~cm}$ below the water surface. On the next day 4, the escape time was recorded. Escape latency (defined as the time required to reach the platform) was the performance index. For gerbils that failed to reach the platform, latency values were recorded at 60 seconds. On the final day, the platform was removed and the number of times that the animals passed through the circular area (passing time with a $10 \mathrm{~cm}$ area) that formerly contained the platform was used as a measure of spatial memory. The probe trial was recorded for 60 seconds.

\section{Histological tests}

\section{GFAP immunofluorescence}

The hippocampus region of gerbil brain tissues were removed and kept in liquid nitrogen. Frozen sections $(5 \mu \mathrm{m})$ containing the hippocampus of gerbils were cut coronally using cryostat. The frozen sections were placed at room temperature and dried. They were fixed at $4{ }^{\circ} \mathrm{C}$ with cold acetone for $10 \mathrm{~min}$. After washing with $0.01 \mathrm{M}$ PBS (Hyclone, USA), $1.2 \%$ hydrogen peroxide was added to incubate for $30 \mathrm{~min}$ to remove nonspecific staining. The sections were washed three times, each time for no less than $10 \mathrm{~min}$, and then the brain sections were blocked with $10 \%$ normal goat serum (Gibco USA) and $0.3 \%$ Triton X-100 (Sigma-Aldrich, USA) in phosphate-buffered saline (PBS) at room temperature for $1 \mathrm{~h}$. Subsequently, mouse brain sections were incubated with rabbit anti-GFAP (1 : 500; Gibco, USA) in $5 \%$ normal goat serum and $0.05 \%$ Triton $X-100$ overnight at $4^{\circ} \mathrm{C}$. After rinsing sections of gerbils in PBS for 3 times, stainings were incubated with secondary antibodies from goat anti-rabbit (Alexa Fluor $^{\circledR}$ 594) (Gibco, USA) in PBS and 0.05\% Triton X-100 for $1 \mathrm{~h}$ at room temperature, rinsed, and mounted in Fluoromount-G (Gibco, USA). Then, sections were stained with $1 \%$ thioflavin $S$ (MedChemExpress MCE, UK) in $80 \%$ ethanol for $15 \mathrm{~min}$ after they were shortly incubated with $70 \%$ ethanol. Subsequently, sections were rinsed in $80 \%$ and $70 \%$ ethanol and distilled water, counterstained with Hoechst, and mounted as described above.
Fluorescent images were obtained at 20x with a fluorescence microscope. For images taken for pixel-based quantification, identical settings were used for all images in a set. For the images chosen for publication, Image J software was applied to remove the distracting artefacts and merge and Photoshop software was used to adjust in to maximize the signal-to-noise ratio, which of relevant features could be seen more clearly. Immunofluorescent analysis was performed to examine the protein distribution of GFAP in the hippocampus region of brains.

\section{Nissl staining}

The hippocampus of gerbil brain tissues were placed in $4 \%$ (w/v) paraformaldehyde (Solarbio, China). After dehydrated by ethanol, the hippocampus tissues were embedded in paraffin blocks and sectioned into $5 \mu \mathrm{m}$ per slice. The gerbil hippocampus sections were incubated for $15 \mathrm{~min}$ at $37^{\circ} \mathrm{C}$ in $0.1 \%$ cresyl violet (Abcam, UK) and then rinsed with demonized water, naturally dried at room temperature, dehydrated with an alcohol gradient, cleared with xylene (Abcam, UK), and mounted with neutral resin. Nissl staining was observed as Nissl bodies found in the cytoplasm of surviving neurons by light microscopy. The integrated optical density/area of the staining in each group was acquired by 2 blinded investigators using Image-Pro Plus 5.1 software.

Gerbil hippocampus sections were rinsed in demonized water and plunged into differentiation liquid for 2 minutes. Sections were dehydrated in ethyl alcohol and sealed by gum. Six hippocampus sections from each animal were analysed.

\section{NeuN immunohistochemistry}

NeuN immunohistochemistry was conducted on $5 \mu \mathrm{m}$-thick hippocampus CA1 sections. After washing in PBS $(3 \times 5 \mathrm{~min})$, tissue sections were processed with $0.3 \% \mathrm{H}_{2} \mathrm{O}_{2}$ for $10 \mathrm{~min}$ and rinsed with PBS. Sections were blocked in $10 \%$ goat plasma for 15 min and labelled with mouse anti-NeuN antibodies (1 : 200; Abcam, UK) overnight at $4^{\circ} \mathrm{C}$. After rinsing, sections were labelled with biocatalytic goat-mouse immunoglobulin G (IgG) (Abcam, UK) for $15 \mathrm{~min}$ at $37^{\circ} \mathrm{C}$. After further washing with PBS, sections were exposed to streptomycin peroxidase (OriGene, USA) for $15 \mathrm{~min}$ at $37^{\circ} \mathrm{C}$. Sections were rinsed in PBS and exposed to 3.3-diaminobenzidine (DAB) for approximately $5 \mathrm{~min}$. Slides were plunged in water to stop the $D A B$ reaction. 


\section{Measurements of malondialdehyde, superoxide dismutase, glutathione and glutathione peroxidase}

The levels of malondialdehyde (MDA) and activity of superoxide dismutase (SOD), glutathione (GSH) and glutathione peroxidase (GSH-PX) were assessed in accordance with the manufacturer's instructions, using commercially available ELISA assay kit (Albino) (Cell Signal, USA). The concentration of MDA was determined through the levels of thiobarbituric-acidreacting substances at a wavelength of $532 \mathrm{~nm}$. MDA was expressed in nanomolars per mg of protein. SOD activity was examined in gerbil hippocampus homogenates through calculation of the rate of inhibition of nucleotide oxidation. Results were expressed as $\mathrm{U} / \mathrm{mg}$ protein. GSH concentrations and GSH-PX activity were assayed through quantification of the rate of oxidation of reduced glutathione to oxidized glutathione by $\mathrm{H}_{2} \mathrm{O}_{2}$. Protein content was dependent upon the mean Coomassie brilliant blue values obtained using bovine plasma albumin as the standard.

\section{Western blot analysis}

Western blotting was performed on hippocampus samples of each of the three groups. Samples were homogenized in ice-cold RIPA buffer and equal aliquots $(50 \mu \mathrm{g})$ of protein were separated by SDS-PAGE electrophoresis and transferred onto nitrocellulose membranes. Membranes were blocked in 5\% nonfat dry milk for $1 \mathrm{~h}$ and probed with anti-caspase 3 ( $1: 1000$; Cell Signal, USA), anti-Bax ( $1: 1000$; Cell Signal, USA), anti-Bcl-2 (1 : 1000; Cell Signal, USA), antiIL-1 (1 : 1000; Cell Signal, USA), anti-IL-6 (1 : 1000; Cell Signal, USA) and anti-TNF- $\alpha$ ( 1 : 1000; Cell Signal, USA) antibodies. Antisera were conjugated to secondary IgG antibodies (1: 5000; Cell Signal, USA). Chemiluminescent detection was performed using standard ECL western blotting detection kit. Band intensities were quantified using BIO-RAD software to obtain the relative protein concentration normalised to the loading control (GAPDH).

\section{ELISA assays}

The levels of secreted IL-1, IL- 6 and TNF- $\alpha$ were determined using a commercially available ELISA kit in accordance with the manufacturer's instructions (Albino). Hippocampus homogenates were collected and IL-1, IL- 6 and TNF- $\alpha$ were measured by ELISA with a microplate reader (epoch, BioTek Instruments, Inc., USA).

\section{Statistical analysis}

All data are presented as the mean \pm SEM. Data were compared using a one-way analysis of variance (ANOVA), and the least significant difference (LSD) post doc analysis was applied for individual comparisons between the three groups means. For all tests, a $p$-value $<0.05$ was regarded as statistically significant $\left({ }^{\#} p\right.$ or ${ }^{\star} p<0.05,{ }^{\# \#} p$ or $\left.{ }^{\star \star} p<0.01\right)$.

\section{Results \\ Lycopene improves spatial learning and memory deficits induced by vascular dementia}

The MWM test was used to observe the effects of Lyc on the spatial learning and memory capability of $\mathrm{VaD}$ gerbil models as shown in Figure 1A. The navigation test indicated a significant decrease in escape latency with the passage of time. This indicates that the gerbils memorized the platform location. When MWM tests were compared across the three groups, we observed statistically significant differences $(p<0.05)$. Escape latency of the $\mathrm{VaD}$ model group at days 2 to 5 of the navigation test was significantly higher than the sham group $(p<0.05)$. This indicates that a learning disability developed in the $\mathrm{VaD}$ model. Compared to the $\mathrm{VaD}$ model group, the $\mathrm{VaD}$ model + Lyc treatment group, which received a Lyc dose of $20 \mathrm{mg} / \mathrm{kg}$, had a significantly lower escape latency of $\mathrm{VaD}$ animals from days 3 to 5 (Fig. $1 \mathrm{~A}$, $p<0.05)$. Importantly, we observed no significant differences between sham and VaD model + Lyc groups which suggests they took time to identify the platform. These results indicated that Lyc improves the learning disability of VaD gerbils.

To confirm these findings, the probe test was performed following navigation assessments. The platform was removed prior to the test and passing shuttle time were used as an indicator of memory capacity (Fig. 1B). Compared with the sham-operated group, VaD model gerbils spent considerably less time in the target area (Fig. 1B, $p<0.05$ ). This indicates that a memory deficit was induced by $\mathrm{VaD}$. In the VaD model + Lyc group, the $\mathrm{VaD}$ gerbils focus on the target quadrant was significantly improved compared to those of the VaD model group, and a significant recovery of passing time was observed (Fig. 1B, 

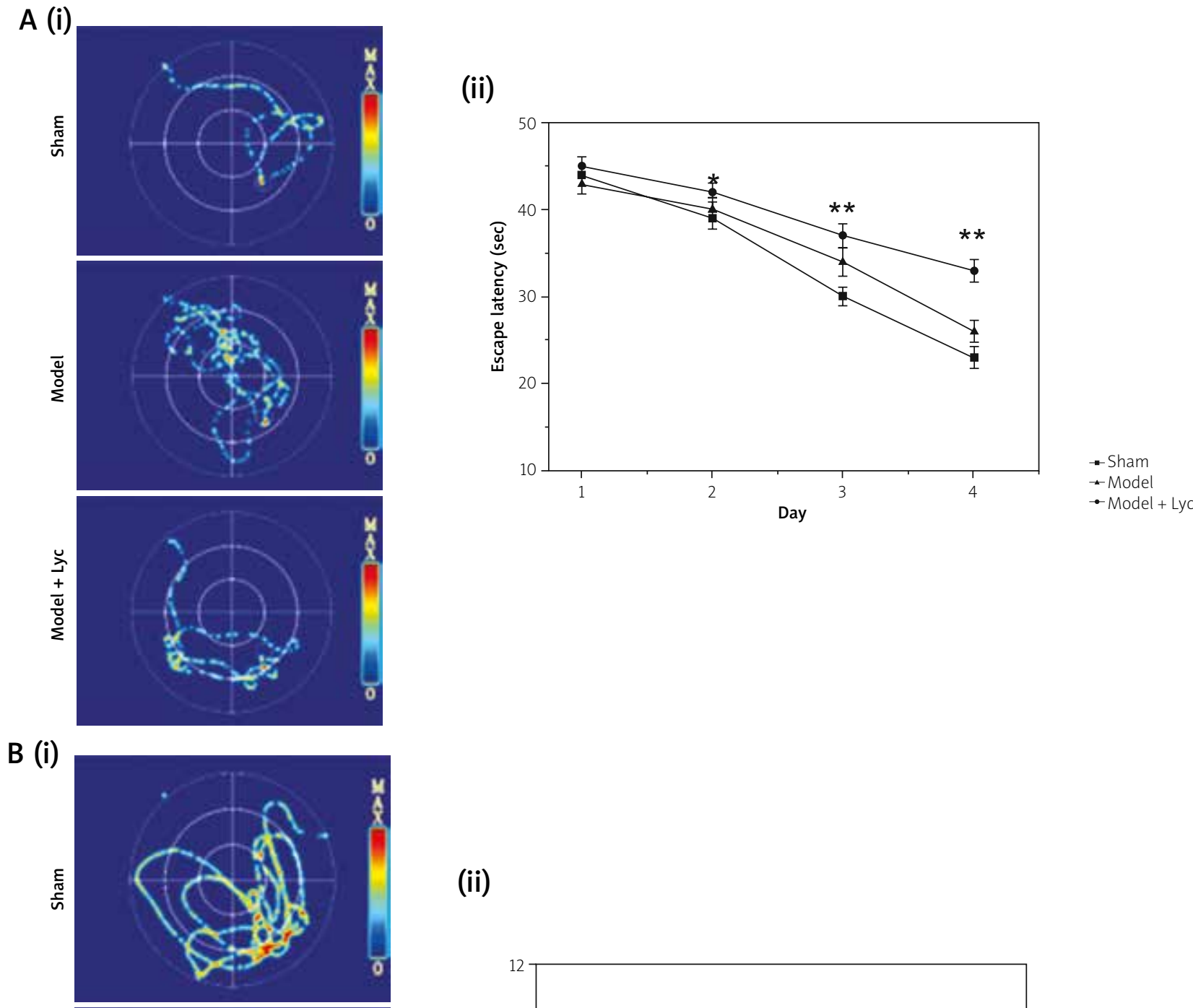

(ii)
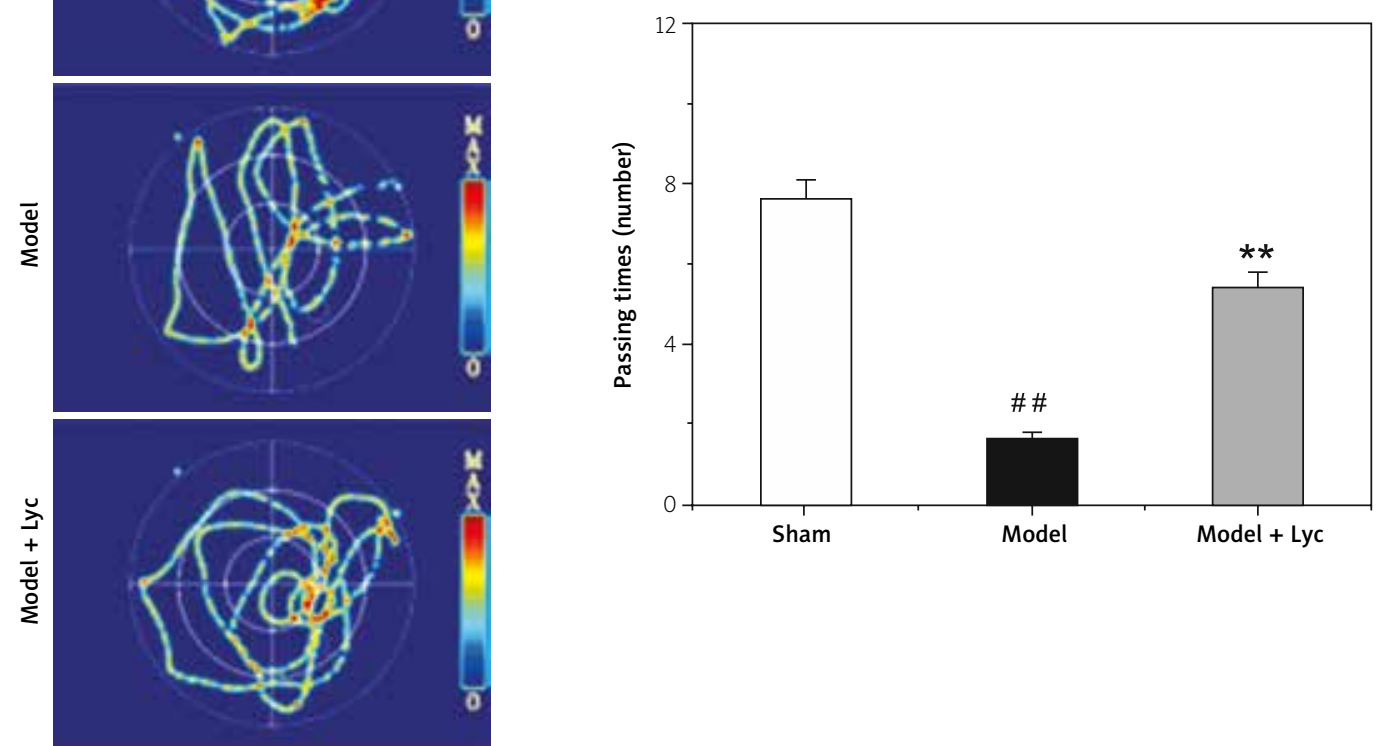

Fig. 1. Lycopene (Lyc) administration prevents VaD-induced learning and memory impairment. A) (i) Representative escape latency images and (ii) average values of latency tests. B) (i) Representative passing time images and (ii) the number of passing times of all animals in each of the three groups. Data are means \pm SEM of gerbils in each group; statistical significance was determined by one-way ANOVA followed by LSD post hoc analysis. ${ }^{\# \#} p<0.01 \mathrm{VaD}$ model vs. Sham; ${ }^{*} p<0.05,{ }^{* *} p<0.01 \mathrm{VaD}$ model + Lyc vs. VaD model. 
A
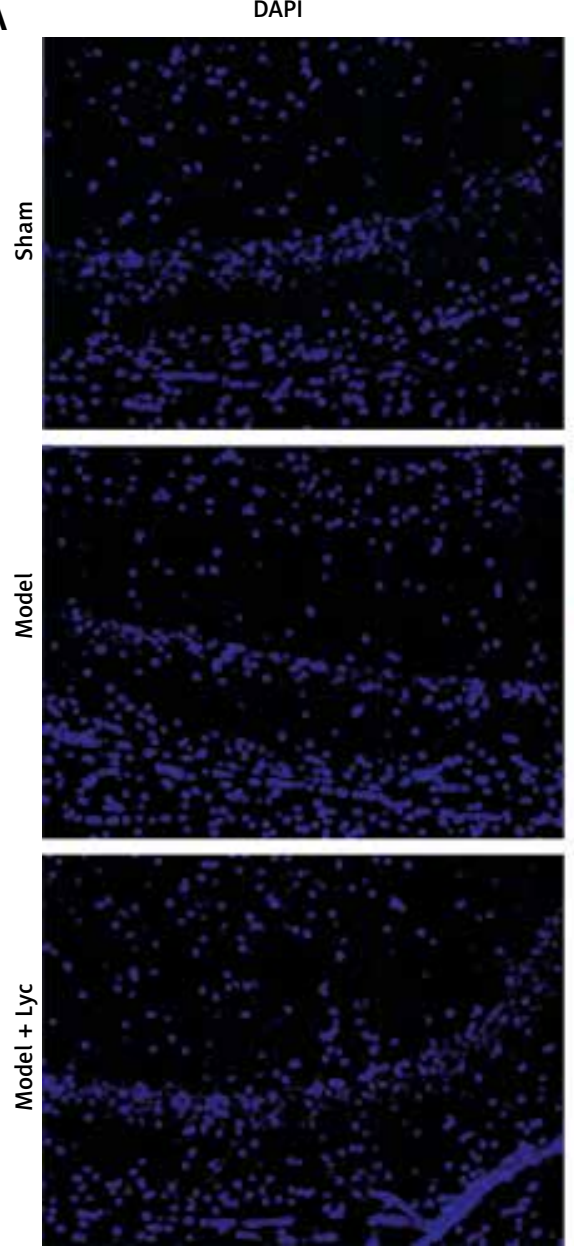

GFAP
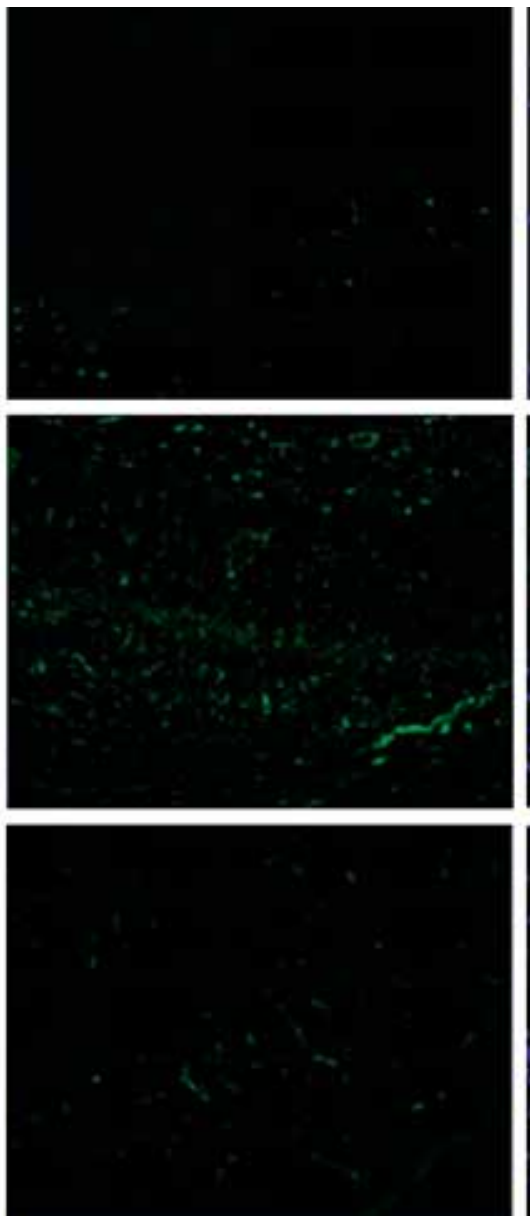

Merge
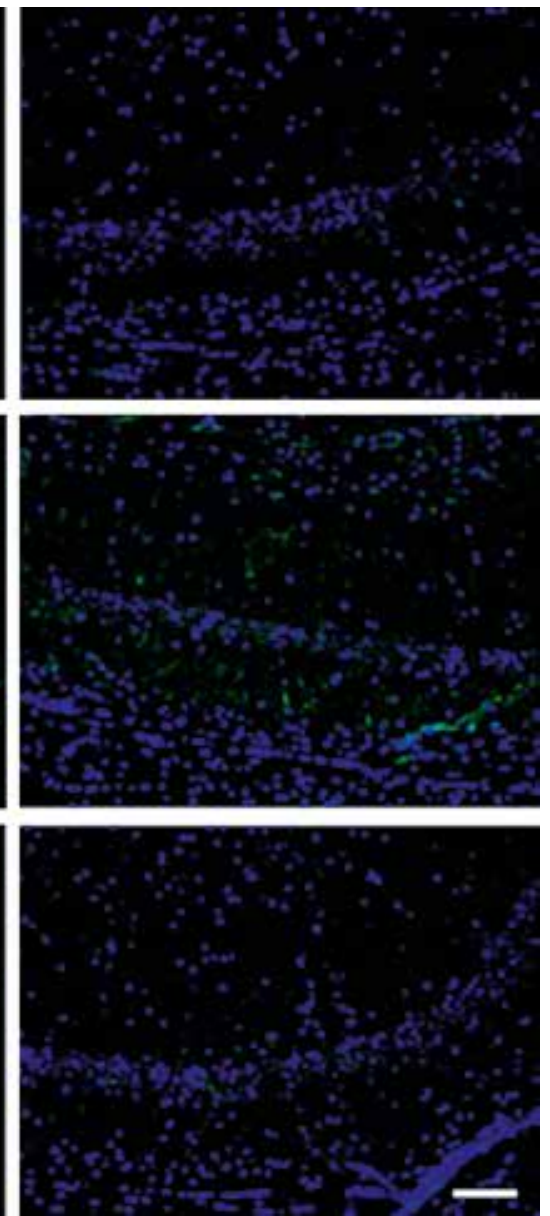

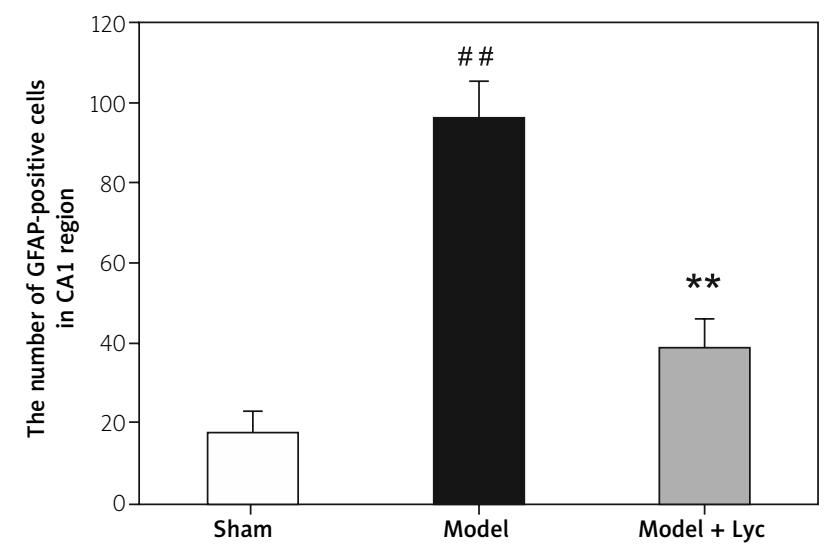

Fig. 2. Lycopene (Lyc) rescued VaD-induced neuronal stress and apoptosis in the hippocampus. A) Representative fluorescent images of DAPI (blue) and GFAP (green) hippocampal slices.

$p<0.05)$. Moreover, we observed no significant differences between Sham groups vs. VaD model + Lyc groups. Taken together, these results indicate that Lyc alleviated the spatial memory deficit induced in the experimental gerbils in VaD.

\section{Histomorphological changes}

Histomorphological changes in hippocampus CA1 regions were characteristic of stress. Sections from the gerbil hippocampus were immunisation for glial fibrillary acidic protein (GFAP), a marker of inter- 

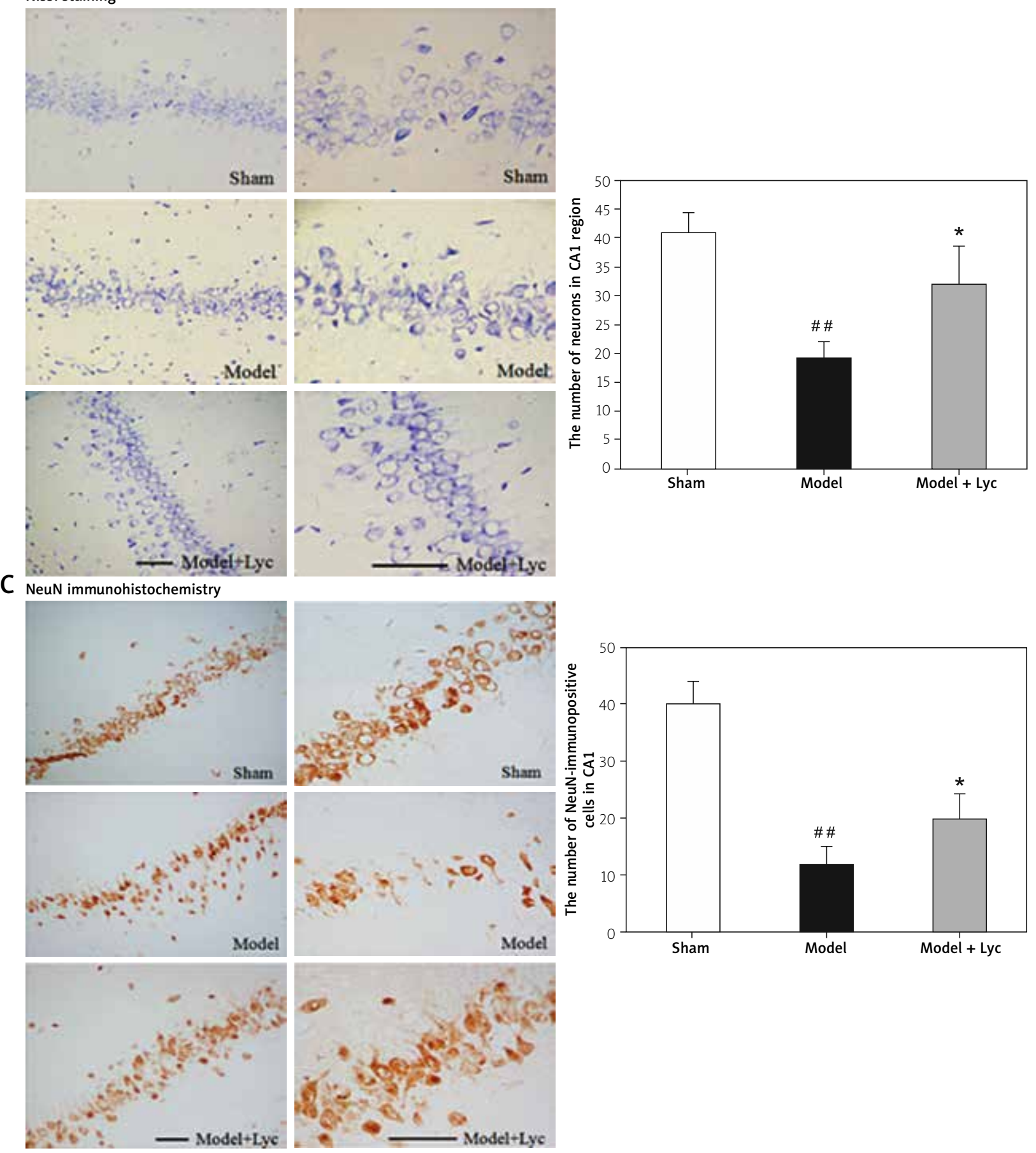

Fig. 2. Cont. B) Photomicrographs of hippocampal CA1 regions with Nissl staining. C) Representative photomicrographs of hippocampal CA1 region with NeuN immunohistochemistry. ${ }^{\#} p<0.01$ VaD model vs. Sham; ${ }^{*} p<0.05,{ }^{* *} p<0.01 \mathrm{VaD}$ model + Lyc vs. VaD model.

mediate filament protein, the expression of which is increased in several situations of brain damage (Fig. 2A). GFAP-immunoreactive astrocytes were found in the stratum radiated, stratum orisons, and stratum laconism-molecule of the CA1 region of all groups. GFAP-immunoreactive astrocytes showed comparable morphology in the hippocampus CA1 region of all gerbil groups, but the numbers of GFAP-immunoreactive astrocytes significantly differed. The number of GFAP-immunoreactive astro- 
cytes in the CA1 region was highest in the $\mathrm{VaD}$ model group (middle panels) and lowest in the sham group (upper panels) consistent with increased brain injury in VaD gerbil models. Importantly, Lyc treatment significantly decreased the number of GFAP immunoreactive cells in $\mathrm{VaD}$ gerbils, producing a comparable GFAP staining pattern of the sham groups (lower panels). This suggested that elevated levels of stress in the brain sub-regions of $\mathrm{VaD}$ mice can be reduced by Lyc treatment.

We further confirmed these effects in hippocampus CA1 sections that were Nissl-stained or marked to NeuN-IR (Fig. 2B, C). A decrease in the number of cells was observed in VaD treated groups compared to sham treated animals. VaD sections stained with Nissl and NeuN revealed many white areas throughout the CA1. However, the number of white areas was reduced to $\mathrm{VaD}$ model + Lyc animals, suggesting that the occurrence of $\mathrm{VaD}$ mediated neurodegeneration is inhibited by Lyc. Through immunotherapy, a qualitative decrease in the number of cells available in the CA1 of VaD treated gerbils was also observed.

Taken together these data suggest that Lyc protects $\mathrm{VaD}$ gerbils from neurodegeneration, explaining its effects on improved cognitive functions in the MWM.

\section{Protective effects of lycopene at the biochemical level}

To determine the molecular mechanisms governing the protection mediated by Lyc for VaD-induced cognitive impairments, we detected hippocampus caspase-3, B-cell lymphoma-2 (Bcl-2), Bcl-2 related protein (Bax), IL-1, IL-6, TNF- $\alpha$ expression in VaD gerbils (Fig. 3). Western blot results showed that expression levels of cleaved caspase- 3 in $\mathrm{VaD}$ model gerbils were significantly higher (0.5-fold increase) than the sham group and $\mathrm{VaD}$ model + Lyc groups (Fig. 3A). The expression levels of Bax (Fig. 3B) and Bcl-2 (Fig. 3C) in the VaD groups were also significantly higher than that in the sham group and $\mathrm{VaD}$ model + Lyc groups. Hippocampus expression levels of IL- 1 and IL-6 in VaD gerbils were also significantly higher than the levels in sham animals, and were reduced by Lyc treatment (Fig. 3D, E). In addition, TNF- $\alpha$ levels in the hippocampus were higher in VaD compared to sham animals. Following Lyc treatment, the levels of TNF- $\alpha$ also decreased (Fig. 3F).

To confirm these findings, we investigated the effects of $\mathrm{VaD}$ treatment on the secretion of IL-1 $\beta$, IL-6, and TNF- $\alpha$ by ELISA. Figure 4 demonstrates that $\mathrm{VaD}$ models displayed significantly higher secretion of all three inflammatory cytokines ( $\geq 5$-fold increase in IL-1 $\beta, \geq 5$-fold increase in IL-6, and $\geq 4$-fold increase in TNF- $\alpha, p<0.05$ ) compared to sham gerbils, the increases of which were significantly inhibited by Lyc treatment (Fig. 4A-C). Taken together, these data suggest $\mathrm{VaD}$ increases inflammatory cytokine expression and causes cell injury in the hippocampus, which can be reversed by Lyc treatment. This may contribute to protective effects of Lyc to VaD-induced cognitive impairment.

\section{Effects of Lyc on iron metabolism and ROS scavengers}

We reasoned that the protective effects of Lyc in $\mathrm{VaD}$ gerbils might be due to the scavenging of free radicals preventing neuronal stress responses, cytokine production and subsequent cell death. To investigate this hypothesis further, we measured the activity of the main free radical scavenging enzyme copper/zinc SOD, GSH that senses and regulates iron levels, and GSH-PX in each treatment group. The effects of $\mathrm{VaD}$ on the activity of these enzymes in the gerbil hippocampus were shown in Figure 5A-C. Significant decreases were found in Mn-SOD, GSH and GSH-PX activity in VaD vs. sham models (2-fold, 3 -fold and 1.8-fold decrease, respectively, $p<0.05$ ). Importantly, the recoveries of SOD, GSH, and GSH-PX activity were shown in gerbils treated by Lyc, which suggested that Lyc acts at the level of ROS scavenging and the regulation of iron metabolism, to minimize VaD mediated neuronal damage.

Malondialdehyde levels were known to be high in the brain during carbonyl stress. To examine the hypothesis that Lyc reverses ROS mediated stress induction mediated by VaD. MDA levels were assessed in all three groups (Fig. 5D). We observed a 2-fold enhanced level of MDA in the brains of VaD gerbils compared to sham gerbils, which were reduced following VaD model + Lyc treatment (1.5-fold reduction following Lyc, $p<0.05)$. Therefore, MDA, as a metabolic product in the brain that weakens cerebral function during carbonyl stress through disrupting the homeostasis between excitatory and inhibitory neurons, is lowered by Lyc treatment in VaD gerbils.

\section{Discussion}

In recent years, neurodevelopmental disorders had increased at a striking rate, primarily due to lifestyle influences and the increasing age of the popula- 
A

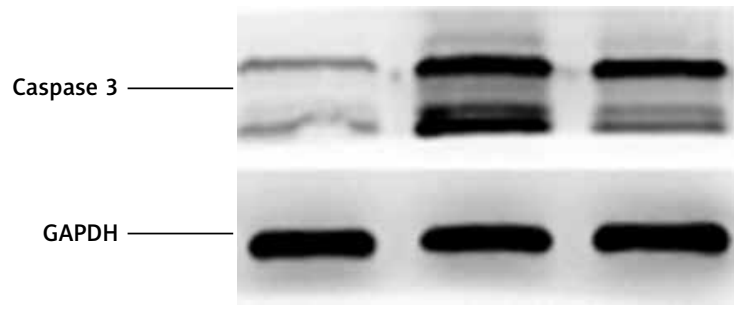

B

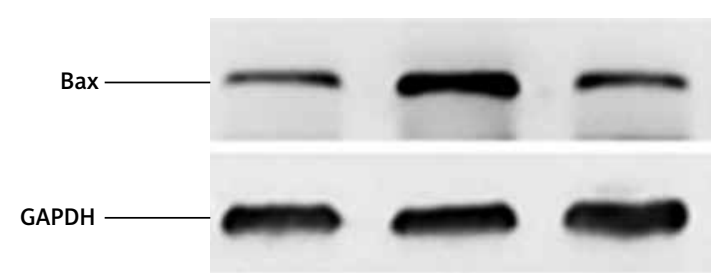

C

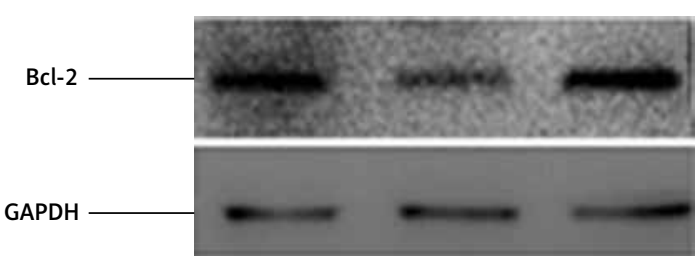

D

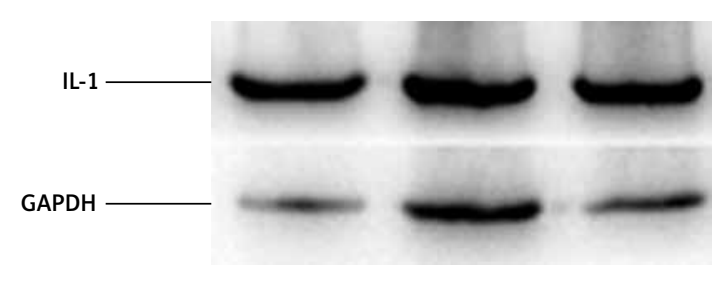

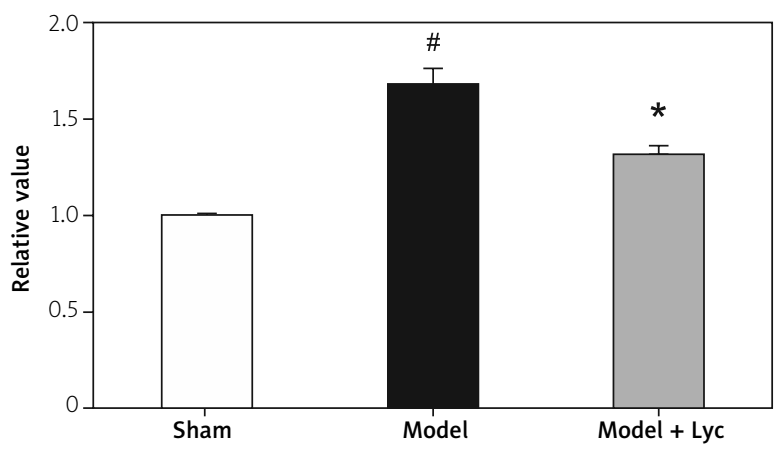
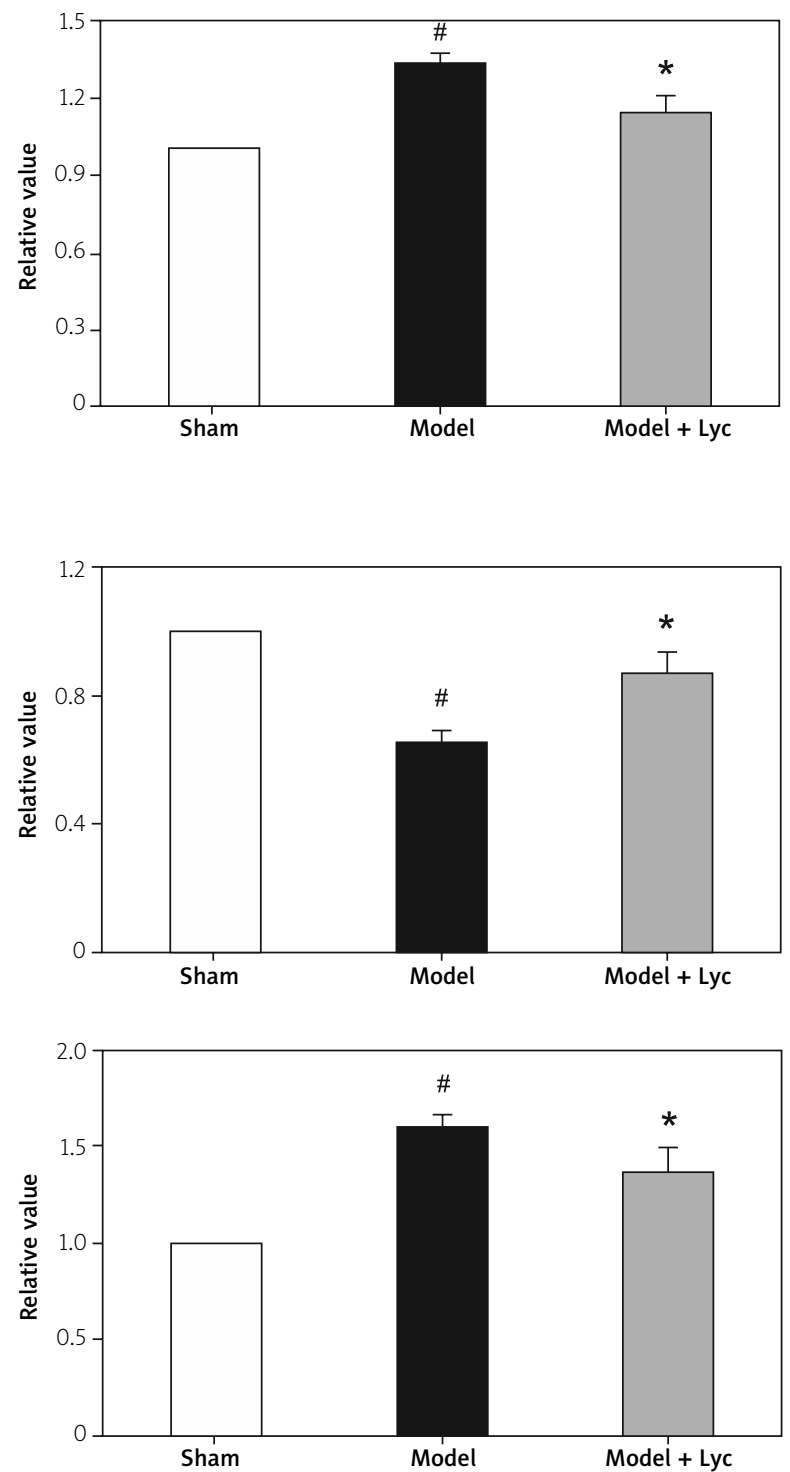

Fig. 3. Lycopene (Lyc) treatment prevents increases in caspase-3, Bax-1, Bcl-2, IL-1, IL-6, and TNF- $\alpha$ in VaD gerbils. A) Representative western blot images and the relative ratio of caspase-3 to GAPDH. B) Western blotting images and the relative ratio of Bax-1 to GAPDH. C) Western blotting images and the relative ratio of Bcl-2-1 to GAPDH. D) Western blotting images and the relative ratio of IL-1 to GAPDH. 
E

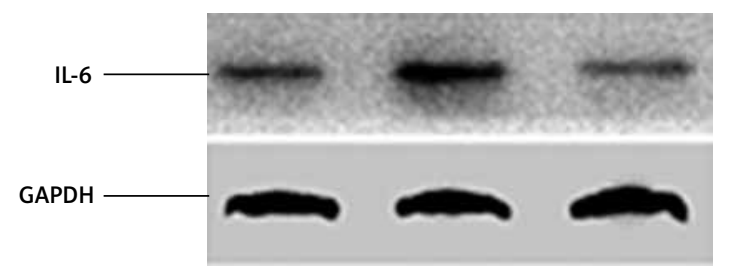

$\mathbf{F}$

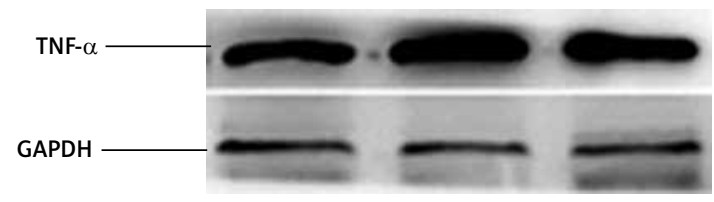

Fig. 3. Cont. E) Western blotting images and the relative ratio of IL- 6 to GAPDH. F) Western blotting images and the relative ratio of TNF- $\alpha$ to GAPDH. Data are the mean \pm SEM of gerbils in each group; statistical significance of difference was determined by one-way ANOVA followed by LSD post hoc analysis. ${ }^{*}<0.05$ VaD model vs. Sham; ${ }^{*} p<0.05$ VaD model + Lyc vs. VaD model.

tion [42]. Alzheimer's disease was the most common form of dementia (60-80\% of all cases) affecting an estimated $\sim 24$ million worldwide, the prevalence of which increases with age. Parkinson's disease (PD) now affects $0.3 \%$ of the global population, up to $1 \%$ of people aged 60 , and up to $3 \%$ of those aged 80 years. $\mathrm{VaD}$ was defined as the impairment of memory and cognitive functioning resulting from CVD, infarcts and leucocytosis [21-23,25,28,29,33]. VaD was considered the second most common cause of cognitive impairment estimated to affect 6-12 per 1,000 people 70 years of age $[39,41,43]$. The mean duration of $\mathrm{VaD}$ was approximately 5 years and its survival rates were lower than $A D$ sufferers and the general population. Given the lack of therapeutic options for $\mathrm{VaD}$ sufferers, this disease represents a global health burden.

Cerebral vascular disease was one of the important causes of senile dementia. Studies had shown
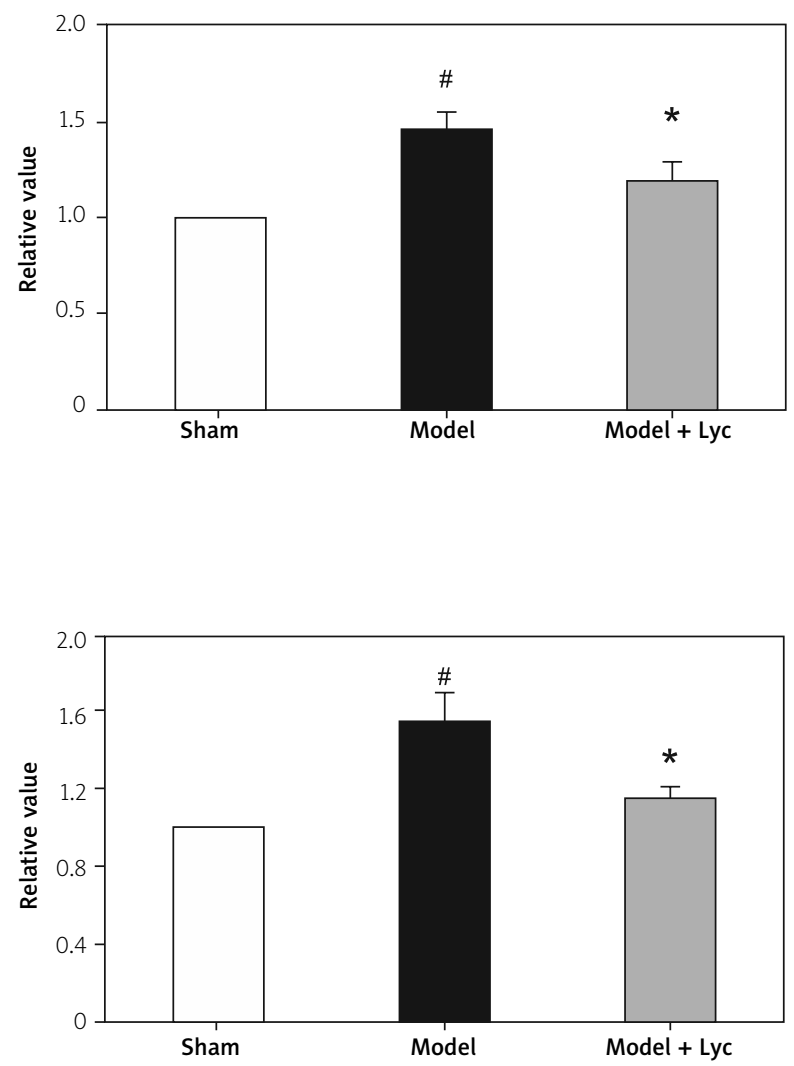

that oxidative stress was closely related to cerebral tissue injury [44]. Recent studies suggested that mitochondrial dysfunction and oxidative stress induction were involved in cognitive decline. With this is in mind, recent work evaluated the beneficial effects of natural antioxidants, including resveratrol-loaded solid lipid antiparticle (R-SLNs) employed to circumvent the problems surrounding brain administration, on VaD [44]. Neurobehavioral analyses in animals administered R-SLNs were promising and cognitive decline was reduced. R-SLN treated rats also displayed a significant reduction in mitochondrial ROS, lipid peroxidation, and protein carbonyls (MDA) [44]. These effects mirror those observed in gerbil models treated with Lyc in this study. In addition, Lyc improved the redox ratio and Mn-SOD activity, also reported for R-SLN.

R-SLNs were also shown to significantly reduce hypoxia-inducible factor- $1 \alpha$ (HIF-1 $\alpha$ ), and increase 

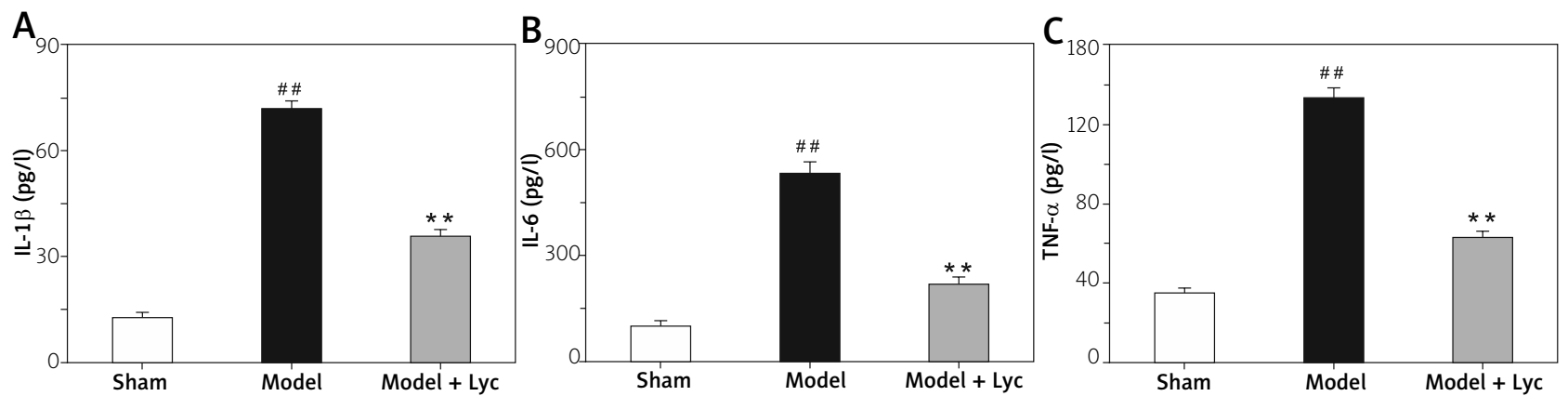

Fig. 4. Levels of A) IL-1 $\beta$, B) IL- 6 and C) TNF- $\alpha$ in the brain of Sham and VaD + Lyc gerbils. Values were obtained by ELISA assay. Data are the mean \pm SEM of gerbils in each group; statistical significance of difference was determined by one-way ANOVA followed by LSD post hoc analysis. ${ }^{\#} p<0.01 \mathrm{VaD}$ model vs. Sham; ${ }^{* *}<0.01$ VaD model + Lyc vs. VaD model.
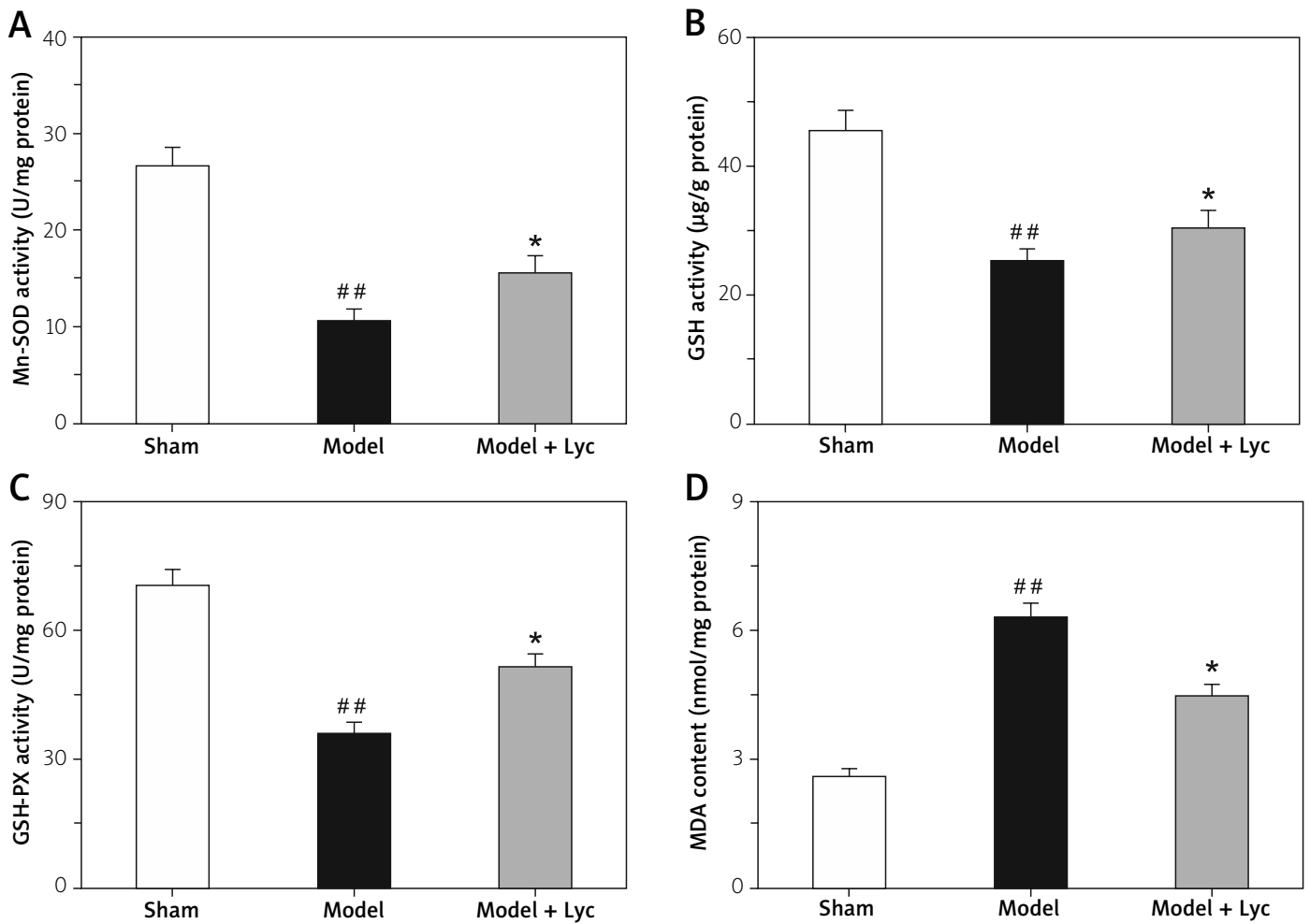

Fig. 5. Lycopene (Lyc) treatment influences the contents of MDA and GSH and the activities of SOD and GSH-PX in hippocampal homogenates of VaD gerbils. A-D) SOD, GSH, GSH-PX and MDA contents or activity in the hippocampus, respectively. Data are expressed as mean \pm SEM of gerbils in each group; statistical significance of difference was determined by one-way ANOVA followed by LSD post hoc analysis. ${ }^{\# \#} p<0.01$ VaD model vs. Sham; ${ }^{*} p<0.05 \mathrm{VaD}$ model + Lyc vs. VaD model.

nuclear factor (erythroid-derived 2)-like 2 (Nrf2) and heme oxygenase 1 (HO-1) levels [44]. Since these factors would be beneficial in an array of age-related neurodegenerative disorders, the influence of Lyc on these hypoxia regulated factors might reveal further benefits to a range of neuronal disorders following Lyc treatment.

In this study, we built the $\mathrm{VaD}$ gerbil models successfully and investigated the effects of the administration of the naturally occurring Lyc on neuronal 
physiological function and cognition in $\mathrm{VaD}$ gerbils. This study showed Lyc could decrease VaD induced hippocampus stress responses and neuronal apoptosis. Furthermore, we found that Lyc was also found to increase the levels of antioxidants including SOD and GSH-PX in brain tissue, and regulate iron metabolism and thus iron mediated neuronal damage through increasing GSH [5,31]. Through these effects, Lyc was able to reduce MDA levels and rescue $\mathrm{VaD}$ induced abnormal learning and memory ability assessed in MWMs. We therefore suggested that Lyc played a protective role by decreasing the levels of pro-apoptotic stimuli, decreasing oxidative stress, and reducing iron mediated neuronal damage, mediating protection against neuronal loss in the hippocampus.

The findings of this study suggest that the consumption of Lyc-rich food may prevent oxidative stress-associated neuronal damage in $\mathrm{VaD}$ and other pathologic conditions. Lyc had been characterized as an inhibitor of ROS induced apoptosis and NF- $\kappa B$, through its effects on calcitonin 1, a known inducer of ROS [27]. Interestingly, serum levels of Lyc as a natural antioxidant pigment were reported to be low in Down syndrome and AD patients, which might be related to their neuronal damage [17]. Whilst this had not been confirmed for $\mathrm{VaD}$, the beneficial effects of Lyc had been demonstrated to suggest a similar scenario. Thus the restoration of Lyc levels in VaD patients represented a viable therapeutic strategy. The use of Lyc as a natural compound had clear benefits over targeted drug development strategies due to their innate affinity for biological receptors, circumventing the side-effects of pharmacological agents $[11,17,27,36]$.

In conclusion, we demonstrated that Lyc increased the learning and memory ability of $\mathrm{VaD}$ gerbils through reducing the pathological changes of the hippocampus, reducing neuronal cell apoptosis. We therefore suggested that enhancing dietary Lyc intake may provide neuroprotection in pathological cases of $\mathrm{VaD}$.

\section{Acknowledgments}

This work was supported by the Scientific Research Strategic Cooperation Project of Nanchong Municipal Government and North Sichuan Medical College (19SXHZ0242), Doctoral Research Initiation Fund of North Sichuan Medical College (CBY17QD02) and the Applied Basic Research Programs of Science and Technology Department of Sichuan Province (2016JY0032).

\section{Disclosure}

The authors report no conflict of interest.

\section{References}

1. Ahern GP, Klyachko VA, Jackson MB. cGMP and S-nitrosylation: two routes for modulation of neuronal excitability by NO. Trends Neurosci 2002; 25: 510-517.

2. Azarpazhooh MR, Avan A, Cipriano LE, Munoz DG, Sposato LA, Hachinski V. Concomitant vascular and neurodegenerative pathologies double the risk of dementia. Alzheimers Dement 2018; 14: 148-156.

3. Baskys A, Hou AC. Vascular dementia: pharmacological treatment approaches and perspectives. Clin Interv Aging 2007; 2: 327-335.

4. Bennett S, Grant MM, Aldred S. Oxidative stress in vascular dementia and Alzheimer's disease: a common pathology. J Alzheimers Dis 2009; 17: 245-257.

5. Berndt C, Lillig CH. Glutathione, glutaredoxins, and iron. Antioxid Redox Signal 2017; 27: 1235-1251.

6. Cho DH, Nakamura T, Fang J, Cieplak P, Godzik A, Gu Z, Lipton SA. S-nitrosylation of Drp1 mediates beta-amyloid-related mitochondrial fission and neuronal injury. Science 2009; 324: 102-105.

7. Dejanovic B, Schwarz G. Neuronal nitric oxide synthase-dependent S-nitrosylation of gephyrin regulates gephyrin clustering at GABAergic synapses. J Neurosci 2014; 34: 7763-7768.

8. Di JH, Li C, Yu HM, Zheng JN, Zhang GY. nNOS downregulation attenuates neuronal apoptosis by inhibiting nNOS-GluR6 interaction and GluR6 nitrosylation in cerebral ischemic reperfusion. Biochem Biophys Res Commun 2012; 420: 594-599.

9. Emdin CA, Rothwell PM, Salimi-Khorshidi G, Kiran A, Conrad N, Callender T, Mehta Z, Pendlebury ST, Anderson SG, Mohseni H, Woodward M, Rahimi K. Blood pressure and risk of vascular dementia: evidence from a primary care registry and a cohort study of transient ischemic attack and stroke. Stroke 2016; 47: 1429-1435.

10. Fang J, Nakamura T, Cho DH, Gu Z, Lipton SA. S-nitrosylation of peroxiredoxin 2 promotes oxidative stress-induced neuronal cell death in Parkinson's disease. Proc Natl Acad Sci U S A 2007; 104: 18742-18747.

11. Fujita K, Yoshimoto N, Kato T, Imada H, Matsumoto G, Inakuma T, Nagata Y, Miyachi E. Lycopene inhibits ischemia/reperfusion-induced neuronal apoptosis in gerbil hippocampal tissue. Neurochem Res 2013; 38: 461-469.

12. Gentil C, Le Guiner C, Falcone S, Hogrel JY, Peccate C, Lorain S, Benkhelifa-Ziyyat S, Guigand L, Montus M, Servais L, Voit T, Pietri-Rouxel F. Dystrophin threshold level necessary for normalization of neuronal nitric oxide synthase, inducible nitric oxide synthase, and ryanodine receptor-calcium release channel type 1 nitrosylation in golden retriever muscular dystrophy dystrophinopathy. Hum Gene Ther 2016; 27: 712-726.

13. Gottesman RF, Schneider AL, Zhou Y, Coresh J, Green E, Gupta N, Knopman DS, Mintz A, Rahmim A, Sharrett AR, Wagenknecht LE, Wong DF, Mosley TH. Association between midlife vascular risk factors and estimated brain amyloid deposition. JAMA 2017; 317: 1443-1450. 
14. Grant WB. Vitamin D might reduce some vascular risk factors and, consequently, risk of dementia. Neth J Med 2011; 69: 51.

15. Gu Z, Kaul M, Yan B, Kridel SJ, Cui J, Strongin A, Smith JW, Lidding ton RC, Lipton SA. S-nitrosylation of matrix metalloproteinases: signaling pathway to neuronal cell death. Science 2002; 297: 1186-1190.

16. Holm H, Nagga K, Nilsson ED, Ricci F, Cinosi E, Melander O, Hansson O, Bachus E, Magnusson M, Fedorowski A. N-terminal prosomatostatin and risk of vascular dementia. Cerebrovasc Dis 2017; 44: 259-265.

17. Hwang S, Lim JW, Kim H. Inhibitory effect of lycopene on amyloid-beta-induced apoptosis in neuronal cells. Nutrients 2017; 9 883.

18. Imfeld P, Bodmer M, Jick SS, Meier CR. Benzodiazepine use and risk of developing Alzheimer's disease or vascular dementia: a case-control analysis. Drug Saf 2015; 38: 909-919.

19. Jaul E, Meiron O. Systemic and disease-specific risk factors in vascular dementia: diagnosis and prevention. Front Aging Neurosc 2017; 9: 333.

20. Jinnouchi H, Kitamura A, Yamagishi K, Kiyama M, Imano H, Okada T, Cui R, Umesawa M, Muraki I, Hayama-Terada M, Kawasaki R, Sankai T, Ohira T, Iso H, Investigators Circs. Retinal vascular changes and prospective risk of disabling dementia: the circulatory risk in communities study (CIRCS). J Atheroscler Thromb 2017; 24: 687 695.

21. Kitagawa K, Miwa K, Okazaki S, Sakaguchi M, Mochizuki H. Serum high-molecular-weight adiponectin level and incident dementia in patients with vascular risk factors. Eur J Neurol 2016; 23: 641-647.

22. Kitagawa K, Miwa K, Yagita Y, Okazaki S, Sakaguchi M, Mochizuki H. Association between carotid stenosis or lacunar infarction and incident dementia in patients with vascular risk factors. Eur I Neurol 2015; 22: 187-192.

23. Kohler S, Buntinx F, Palmer K, van den Akker M. Depression, vascular factors, and risk of dementia in primary care: a retrospective cohort study. J Am Geriatr Soc 2015; 63: 692-698.

24. Koseoglu E, Karaman Y. Relations between homocysteine, folate and vitamin B12 in vascular dementia and in Alzheimer disease. Clin Biochem 2007; 40: 859-863.

25. Larson EB, ACP Journal Club. Review: In older persons at vascular risk, statins do not prevent dementia or cognitive decline at 3.5 to 5 years. Ann Intern Med 2016; 164: JC42.

26. Li P, Zhu ML, Pan GP, Lu JX, Zhao FR, Jian X, Liu LY, Wan GR, Chen Y, Ping S, Wang SX, Hu CP. Vitamin B6 prevents isocarbophos-induced vascular dementia in rats through $\mathrm{N}$-methyl-D-aspartate receptor signaling. Clin Exp Hypertens 2018; 40: 192-201.

27. Lim S, Hwang S, Yu JH, Lim JW, Kim H. Lycopene inhibits regulator of calcineurin 1-mediated apoptosis by reducing oxidative stress and down-regulating Nucling in neuronal cells. Mol Nutr Food Res 2017; 61.

28. Lin WC, Hu LY, Tsai SJ, Yang AC, Shen CC. Depression and the risk of vascular dementia: a population-based retrospective cohort study. Int J Geriatr Psychiatry 2017; 32: 556-563.

29. Mikkola TS, Savolainen-Peltonen H, Tuomikoski P, Hoti F, Vattulainen P, Gissler M, Ylikorkala O. Lower death risk for vascular dementia than for Alzheimer's disease with postmenopausal hormone therapy users. J Clin Endocrinol Metab 2017; 102: 870-877.

30. Miwa K, Tanaka M, Okazaki S, Yagita Y, Sakaguchi M, Mochizuki H, Kitagawa K. Increased total homocysteine levels predict the risk of incident dementia independent of cerebral small-vessel diseases and vascular risk factors. J Alzheimers Dis 2016; 49: 503-513.

31. Yeonsil M, Han SH, Moon WJ. Patterns of brain iron accumulation in vascular dementia and alzheimer's dementia using quantitative susceptibility mapping imaging. J Alzheimers Dis 2016; 51: 737-745.

32. Moretti R, Caruso P, Dal Ben M, Conti C, Gazzin S, Tiribelli C. Vita$\min \mathrm{D}$, homocysteine, and folate in subcortical vascular dementia and alzheimer dementia. Front Aging Neurosci 2017; 9: 169.

33. Portegies ML, Wolters FJ, Hofman A, Ikram MK, Koudstaal PJ, Ikram MA. Prestroke vascular pathology and the risk of recurrent stroke and poststroke dementia. Stroke 2016; 47: 2119-2122.

34. Prabhakar P, Chandra SR, Supriya M, Issac TG, Prasad C, Christopher R. Vitamin D status and vascular dementia due to cerebral small vessel disease in the elderly Asian Indian population. J Neurol Sci 2015; 359: 108-111.

35. Quadri P, Fragiacomo C, Pezzati R, Zanda E, Forloni G, Tettamanti M, Lucca U. Homocysteine, folate, and vitamin B-12 in mild cognitive impairment, Alzheimer disease, and vascular dementia. Am J Clin Nutr 2004; 80: 114-122.

36. Sachdeva AK, Chopra K. Lycopene abrogates Abeta(1-42)-mediated neuroinflammatory cascade in an experimental model of Alzheimer's disease. J Nutr Biochem 2015; 26: 736-744.

37. Serra JA, Dominguez RO, de Lustig ES, Guareschi EM, Famulari AL, Bartolome EL, Marschoff ER. Parkinson's disease is associated with oxidative stress: comparison of peripheral antioxidant profiles in living Parkinson's, Alzheimer's and vascular dementia patients. J Neural Transm (Vienna) 2001; 108: 1135-1148.

38. Shi GX, Liu CZ, Wang LP, Guan LP, Li SQ. Biomarkers of oxidative stress in vascular dementia patients. Can J Neurol Sci 2012; 39: 65-68.

39. Sorrentino G, Migliaccio R, Bonavita V. Treatment of vascular dementia: the route of prevention. Eur Neurol 2008; 60: 217-223.

40. Stephan BCM, Minett T, Muniz-Terrera G, Harrison SL, Matthews FE, Brayne C. Neuropsychological profiles of vascular disease and risk of dementia: implications for defining vascular cognitive impairment no dementia (VCI-ND). Age Ageing 2017; 46: 755-760.

41. Tariq S, Barber PA. Dementia risk and prevention by targeting modifiable vascular risk factors. J Neurochem 2018; 144: 565-581.

42. Teijido O, Carril JC, Cacabelos R. Population-based study of risk polymorphisms associated with vascular disorders and dementia. Curr Genomics 2017; 18: 430-441.

43. Ueno M. Elucidation of mechanism of blood-brain barrier damage for prevention and treatment of vascular dementia. Rinsho Shinkeigaku 2017; 57: 95-109.

44. Yadav A, Sunkaria A, Singhal N, Sandhir R. Resveratrol loaded solid lipid nanoparticles attenuate mitochondrial oxidative stress in vascular dementia by activating Nrf2/HO-1 pathway. Neurochem Int 2018; 112: 239-254.

45. Zhang J, Guo W, Tian B, Sun M, Li H, Zhou L, Liu X. Puerarin attenuates cognitive dysfunction and oxidative stress in vascular dementia rats induced by chronic ischemia. Int I Clin Exp Pathol 2015; 8: 4695-4704.

46. Zhao RR, Xu F, Xu XC, Tan GJ, Liu LM, Wu N, Zhang WZ, Liu JX. Effects of alpha-lipoic acid on spatial learning and memory, oxidative stress, and central cholinergic system in a rat model of vascular dementia. Neurosci Lett 2015; 587: 113-119. 